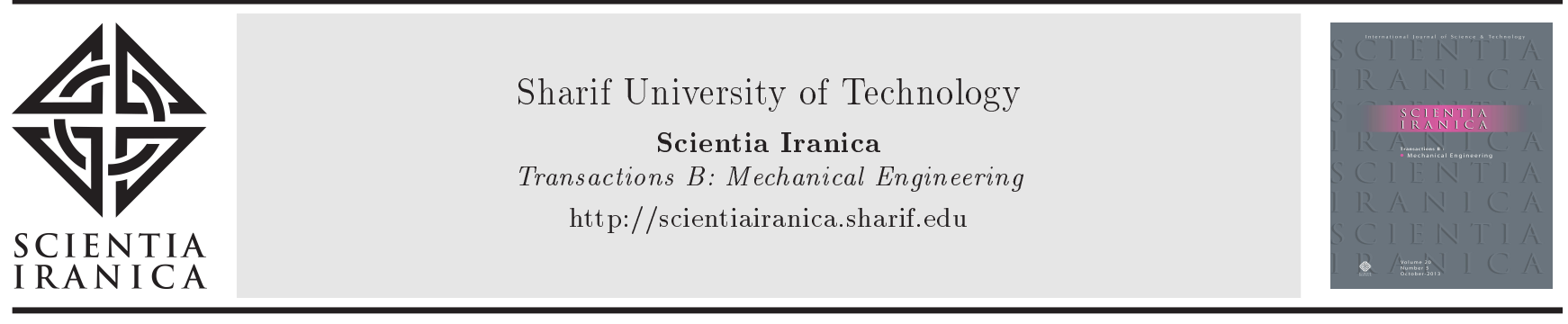

\title{
Computational analysis of shallow water waves with Korteweg-de Vries equation
}

\author{
T. Ak ${ }^{a, *}$ H. Triki ${ }^{\mathrm{b}}$, S. Dhawan ${ }^{\mathrm{c}}$, S.K. Bhowmik ${ }^{\mathrm{d}}$, S.P. Moshokoa ${ }^{\mathrm{e}}$, \\ M.Z. Ullah ${ }^{\mathrm{f}}$, and A. Biswas ${ }^{\mathrm{e}, \mathrm{f}}$ \\ a. Department of Transportation Engineering, Yalova University, Yalova 77100, Turkey. \\ b. Radiation Physics Laboratory, Department of Physics, Faculty of Sciences, Badji Mokhtar University, P.O. Box 12, 23000 \\ Annaba, Algeria. \\ c. Department of Mathematics, Central University of Haryana, Haryana 123029, India. \\ d. Department of Mathematics, University of Dhaka, Dhaka 1000, Bangladesh. \\ e. Department of Mathematics and Statistics, Tshwane University of Technology, Pretoria-0008, South Africa. \\ f. Department of Mathematics, College of Science, King Khalid University, P.O. Box 9004, Abha-61413, Saudi Arabia.
}

Received 13 November 2016; received in revised form 6 August 2017; accepted 2 October 2017

\section{KEYWORDS \\ gKdV equation; \\ Finite element \\ method; \\ Collocation; \\ Shallow water; \\ B-spline.}

\begin{abstract}
The collocation finite element method was applied to obtain solitary wave solutions to Korteweg-de Vries equation with power law nonlinearity. The stability and error analyses were also carried out for these waves. Additionally, conservation laws were studied numerically.
\end{abstract}

(C) 2018 Sharif University of Technology. All rights reserved.

\section{Introduction}

The dynamics of shallow water waves has been an active research area during the past several decades. In this context, there are several models that govern this wave flow. A few of these commonly studied models are Boussinesq equation [1], Kawahara equation [2], Peregrine equation [3], Benjamin-Bona-Mahoney equation [4], and several others [5-8]. This paper studies the model with the aid of Korteweg-de Vries (KdV) equation. In order to keep this on a generalized setting, power law nonlinearity is considered; note that the $\mathrm{KdV}$ equation and modified $\mathrm{KdV}$ equation become special cases.

There are several analytical and numerical schemes that are applied to understand the shal-

*. Corresponding author.

E-mail address: turgut.ak@yalova.edu.tr (T. Ak)

doi: $10.24200 /$ sci.2017.4518 low water wave phenomena [9-13]. Some of these algorithms are exp-function method, $G^{\prime} / G$ expansion scheme $[14,15]$, method of undetermined coefficients, semi-inverse variational principle, Galerkin method [16,17], Petrov-Galerkin method [18], collocation method [19-21], and several others [22-24]. Also, the solutions to some fractional differential equations have been discussed $[25,26]$. This paper studies power law $\mathrm{KdV}$ equation by using collocation finite element method. The stability analysis as well as conservation laws are both addressed.

The well-known Korteweg-de Vries (KdV) equation:

$$
U_{t}+\varepsilon U U_{x}+\mu U_{x x x}=0
$$

where $\varepsilon$ and $\mu$ are the nonlinear and dispersion coefficients, respectively, is the generic model for the study of weakly nonlinear long waves [27]. It arises in physical systems, which involve a balance between nonlinearity and dispersion at leading-order [28]. For example, it describes surface waves of long wavelength 
and small amplitude on shallow water and internal waves in a shallow density-stratified fluid [28]. This nonlinear wave equation has been studied extensively by numerical methods [29-35].

The generalized $\mathrm{KdV}$ equation with power law nonlinearity is given by:

$$
U_{t}+\varepsilon U^{p} U_{x}+\mu U_{x x x}=0,
$$

with $p \in \mathbb{R}$. In particular, the case $p=1$ leads to the regular $\mathrm{KdV}$ equation (1). Here, the first term is the evolution term, while the second term represents the nonlinear term and the third term is the dispersion term. As value of $p$ increases, the solitary wave breaks down. The solitary waves blow up at $p=4[36,37]$.

In this paper, the collocation finite element method based on quintic B-spline functions is successfully applied to the generalized $\mathrm{KdV}$ equation to examine the motion of a single solitary wave whose analytical solution is known. Also, evolution of solitons is studied with Gaussian and undular bore initial conditions.

\section{Governing equation and quintic B-spline basis functions}

In this study, we consider the generalized Kortewegde Vries (gKdV) equation with the physical boundary conditions $U \rightarrow 0$ as $x \rightarrow \pm \infty$, where $\varepsilon$ and $\mu$ are positive parameters and the subscripts $x$ and $t$ denote the differentiation. To implement the numerical method, solution domain is restricted to an interval $a \leq x \leq b$. Boundary conditions will be selected from the following homogeneous boundary conditions:

$$
U_{N}(a, t)=0, \quad U_{N}(b, t)=0,
$$

$$
\left(U_{N}\right)_{x}(a, t)=0, \quad\left(U_{N}\right)_{x}(b, t)=0, \quad t>0,
$$

and the initial condition:

$$
U(x, 0)=f(x) \quad a \leq x \leq b .
$$

The quintic B-splines $\phi_{m}(x)(m=-2(1) N+2)$ at the knots $x_{m}$ are defined over the interval $[a, b]$ by the relationships [38] shown in Box I. The set of functions $\left\{\phi_{-2}(x), \phi_{-1}(x), \phi_{0}(x), \cdots, \phi_{N+1}(x), \phi_{N+2}(x)\right\}$ forms a basis for functions defined over $[a, b]$. The approximate solution $U_{N}(x, t)$ to the exact solution $U(x, t)$ is given by:

$$
U_{N}(x, t)=\sum_{j=-2}^{N+2} \phi_{j}(x) \delta_{j}(t),
$$

where $\delta_{j}(t)$ are time dependent parameters to be determined from the boundary and collocation conditions. Each quintic B-spline covers six elements so that each element $\left[x_{m}, x_{m+1}\right]$ is covered by six splines. The values of $\phi_{m}(x)$ and its derivative may be tabulated as in Table 1 .

Using trial function (Eq. (6)) and quintic Bsplines (Eq. (5)), the values of $U, U^{\prime}, U^{\prime \prime}, U^{\prime \prime \prime}$ and $U^{i v}$, at the knots are determined in terms of the element parameters $\delta_{m}$ by:

$$
\begin{aligned}
U_{m}= & U\left(x_{m}\right)=\delta_{m-2}+26 \delta_{m-1}+66 \delta_{m}+26 \delta_{m+1} \\
& +\delta_{m+2}, \\
U_{m}^{\prime}= & U^{\prime}\left(x_{m}\right)=\frac{5}{h}\left(-\delta_{m-2}-10 \delta_{m-1}+10 \delta_{m+1}\right. \\
& \left.+\delta_{m+2}\right)
\end{aligned}
$$

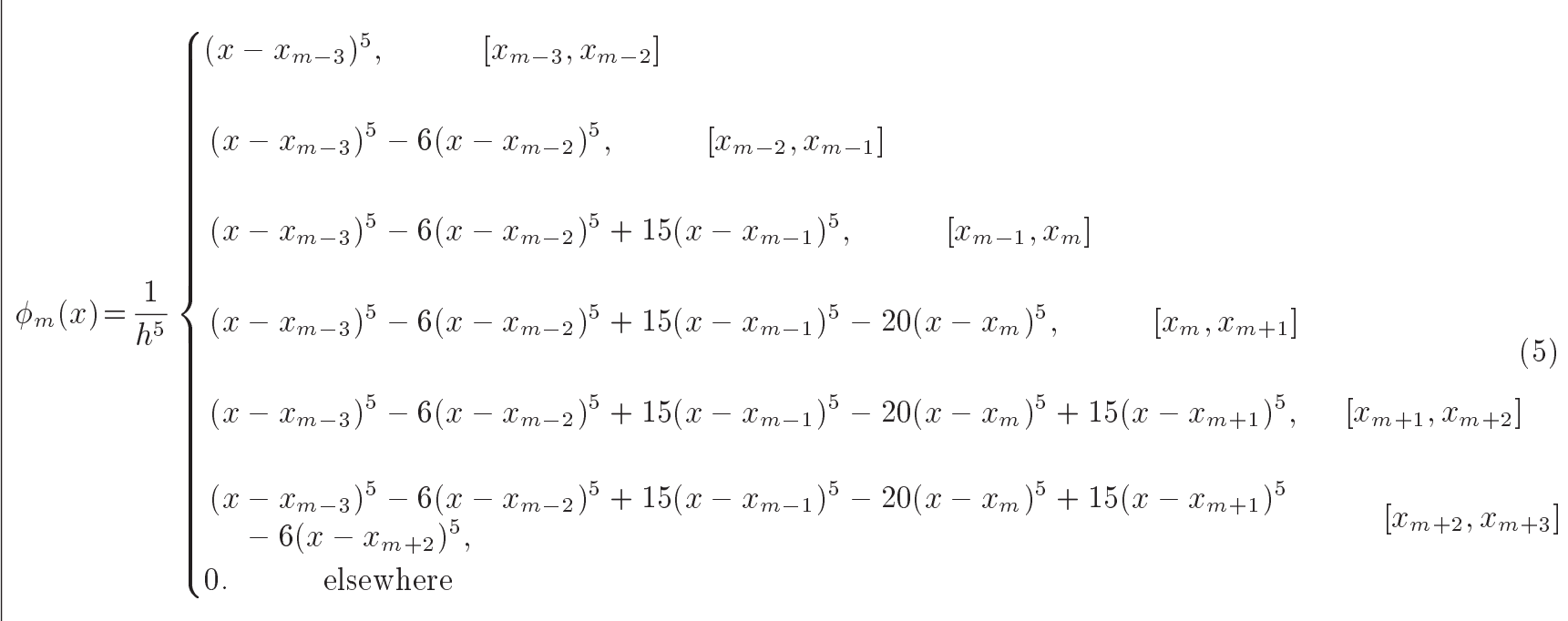


Table 1. Quintic B-spline function and its derivatives at nodes $x_{m}$.

\begin{tabular}{lccccccc}
\hline \multicolumn{1}{c}{$\boldsymbol{x}$} & $\boldsymbol{x}_{\boldsymbol{m}-\mathbf{3}}$ & $\boldsymbol{x}_{\boldsymbol{m}-\mathbf{2}}$ & $\boldsymbol{x}_{\boldsymbol{m}-\mathbf{1}}$ & $\boldsymbol{x}_{\boldsymbol{m}}$ & $\boldsymbol{x}_{\boldsymbol{m}+\mathbf{1}}$ & $\boldsymbol{x}_{\boldsymbol{m}+\mathbf{2}}$ & $\boldsymbol{x}_{\boldsymbol{m}+\mathbf{3}}$ \\
\hline$\phi_{m}(x)$ & 0 & 1 & 26 & 66 & 26 & 1 & 0 \\
$h \phi_{m}^{\prime}(x)$ & 0 & -5 & -50 & 0 & 50 & 5 & 0 \\
$h^{2} \phi_{m}^{\prime \prime}(x)$ & 0 & 20 & 40 & -120 & 40 & 20 & 0 \\
$h^{3} \phi_{m}^{\prime \prime \prime}(x)$ & 0 & -60 & 120 & 0 & -120 & 60 & 0 \\
$h^{4} \phi_{m}^{i v}(x)$ & 0 & 120 & -480 & 720 & -480 & 120 & 0 \\
\hline
\end{tabular}

$$
\begin{aligned}
U_{m}^{\prime \prime}= & U^{\prime \prime}\left(x_{m}\right)=\frac{20}{h^{2}}\left(\delta_{m-2}+2 \delta_{m-1}-6 \delta_{m}\right. \\
& \left.+2 \delta_{m+1}+\delta_{m+2}\right), \\
U_{m}^{\prime \prime \prime}= & U^{\prime \prime \prime}\left(x_{m}\right)=\frac{60}{h^{3}}\left(-\delta_{m-2}+2 \delta_{m-1}-2 \delta_{m+1}\right. \\
& \left.+\delta_{m+2}\right), \\
U_{m}^{i v}= & U^{i v}\left(x_{m}\right)=\frac{120}{h^{4}}\left(\delta_{m-2}-4 \delta_{m-1}+6 \delta_{m}\right. \\
& \left.-4 \delta_{m+1}+\delta_{m+2}\right),
\end{aligned}
$$

where the symbols ', ", '"', and iv denote the first, second, third, and fourth differentiation with respect to $x$, respectively. The splines $\phi_{m}(x)$ and their four principal derivatives vanish outside the interval $\left[x_{m-3}, x_{m+3}\right]$.

\section{Collocation finite element method}

Using the nodal values of $U_{m}$ and its spatial derivatives given by Eq. (7) in Eq. (2), the following general form of the solution approach is obtained for the linearization technique:

$$
\begin{aligned}
\dot{\delta}_{m-2} & +26 \dot{\delta}_{m-1}+66 \dot{\delta}_{m}+26 \dot{\delta}_{m+1}+\dot{\delta}_{m+2} \\
& +\frac{5 \varepsilon Z_{m}}{h}\left(-\delta_{m-2}-10 \delta_{m-1}+10 \delta_{m+1}+\delta_{m+2}\right) \\
& +\frac{60 \mu}{h^{3}}\left(-\delta_{m-2}+2 \delta_{m-1}-2 \delta_{m+1}+\delta_{m+2}\right)=0
\end{aligned}
$$

where:

$$
\begin{aligned}
Z_{m}= & U_{m}^{p}=\left(\delta_{m-2}+26 \delta_{m-1}+66 \delta_{m}+26 \delta_{m+1}\right. \\
& \left.+\delta_{m+2}\right)^{p}
\end{aligned}
$$

and "." indicates derivative with respect to $t$. For the linearization technique, the term $U^{p}$ in non-linear term $U^{p} U_{x}$ is taken as Eq. (9) by assuming that the quantity $U^{p}$ is locally constant.

If time parameters $\delta_{i}$ and their time derivatives $\dot{\delta}_{i}$ in Eq. (8) are discretized by the Crank-Nicolson formula and usual finite difference approximation, respectively:

$$
\delta_{i}=\frac{\delta_{i}^{n+1}+\delta_{i}^{n}}{2}, \quad \dot{\delta}_{i}=\frac{\delta_{i}^{n+1}-\delta_{i}^{n}}{\Delta t}
$$

We obtain a recurrence relationship between two time levels $n$ and $n+1$ relating two unknown parameters in $\delta_{i}^{n+1}$ and $\delta_{i}^{n}$ for $i=m-2, m-1, \cdots, m+1, m+2$ :

$$
\begin{aligned}
\gamma_{1} \delta_{m-2}^{n+1} & +\gamma_{2} \delta_{m-1}^{n+1}+\gamma_{3} \delta_{m}^{n+1}+\gamma_{4} \delta_{m+1}^{n+1}+\gamma_{5} \delta_{m+2}^{n+1} \\
= & \gamma_{5} \delta_{m-2}^{n}+\gamma_{4} \delta_{m-1}^{n}+\gamma_{3} \delta_{m}^{n}+\gamma_{2} \delta_{m+1}^{n} \\
& +\gamma_{1} \delta_{m+2}^{n}
\end{aligned}
$$

where:

$$
\begin{aligned}
& \gamma_{1}=\left[1-E Z_{m}-M\right], \\
& \gamma_{2}=\left[26-10 E Z_{m}+2 M\right], \\
& \gamma_{3}=[66], \\
& \gamma_{4}=\left[26+10 E Z_{m}-2 M\right], \\
& \gamma_{5}=\left[1+E Z_{m}+M\right], \\
& m=0,1, \cdots, N, \quad M=\frac{30}{h^{3}} \mu \Delta t . \\
& E=\frac{5}{2 h} \varepsilon \Delta t, \quad .
\end{aligned}
$$

The system (11) consists of $(N+1)$ linear equations including $(N+5)$ unknown parameters $\left(\delta_{-2}, \delta_{-1}, \cdots, \delta_{N+1}, \delta_{N+2}\right)^{T}$. To obtain a unique solution to this system, we need four additional constraints. They are obtained from the boundary conditions and can be used to eliminate $\delta_{-2}, \delta_{-1}$ and $\delta_{N+1}, \delta_{N+2}$ from the system in Eq. (11), which later becomes a matrix equation for the $N+1$ unknowns $d=\left(\delta_{0}, \delta_{1}, \cdots, \delta_{N}\right)^{T}$ of the form:

$$
A \mathbf{d}^{\mathbf{n}+\mathbf{1}}=B \mathbf{d}^{\mathbf{n}}
$$

The matrices $A$ and $B$ are pentagonal $(N+1) \times(N+1)$ and can be solved by using the pentagonal algorithm. However, two or three inner iterations are implemented for the term $\delta^{n *}=\delta^{n}+\frac{1}{2}\left(\delta^{n}-\delta^{n-1}\right)$ at each time step to cope with the non-linearity caused by $Z_{m}$. Before the solution process begins iteratively, the initial vector 
$\mathbf{d}^{\mathbf{0}}=\left(\delta_{0}, \delta_{1}, \cdots, \delta_{N-1}, \delta_{N}\right)$ must be determined by using the initial condition and the following derivatives at the boundary conditions:

$$
\begin{array}{ll}
U_{N}(x, 0)=U\left(x_{m}, 0\right), & m=0,1,2, \cdots, N, \\
\left(U_{N}\right)_{x}(a, 0)=0, & \left(U_{N}\right)_{x}(b, 0)=0, \\
\left(U_{N}\right)_{x x}(a, 0)=0, & \left(U_{N}\right)_{x x}(b, 0)=0 .
\end{array}
$$

Thus, we have the following matrix form of the initial vector $\mathbf{d}^{\mathbf{0}}$ :

$$
W \mathbf{d}^{\mathbf{0}}=C,
$$

where:

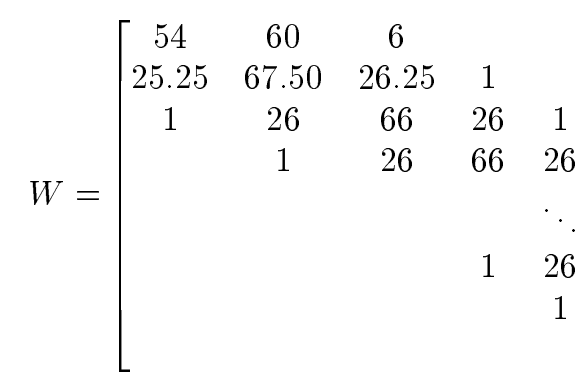

$$
\left.\begin{array}{ccc} 
& & \\
1 & & \\
66 & 26 & 1 \\
26.25 & 67.50 & 25.25 \\
6 & 60 & 54
\end{array}\right],
$$

and:

$$
C=\left[U\left(x_{0}, 0\right), U\left(x_{1}, 0\right), \cdots, U\left(x_{N-1}, 0\right), U\left(x_{N}, 0\right)\right]^{T} .
$$

This matrix system can be solved be efficiently by using a variant of Thomas algorithm.

\section{Stability analysis}

The stability analysis is based on the Von Neumann theory. The growth factor $\xi$ of the error in a typical mode of amplitude $\xi^{n}$ is:

$$
\delta_{m}^{n}=\xi^{n} e^{i m k h}
$$

where $k$ is the mode number and $h$, the element size, is determined by linearization of the numerical scheme. Substituting the Fourier mode (Eq. (19)) into Eq. (11) gives the following equality:

$$
\begin{aligned}
\gamma_{1} \xi^{n+1} & e^{i(m-2) k h}+\gamma_{2} \xi^{n+1} e^{i(m-1) k h}+\gamma_{3} \xi^{n+1} e^{i m k h} \\
& +\gamma_{4} \xi^{n+1} e^{i(m+1) k h}+\gamma_{5} \xi^{n+1} e^{i(m+2) k h} \\
= & \gamma_{5} \xi^{n} e^{i(m-2) k h}+\gamma_{4} \xi^{n} e^{i(m-1) k h}+\gamma_{3} \xi^{n} e^{i m k h} \\
& +\gamma_{2} \xi^{n} e^{i(m+1) k h}+\gamma_{1} \xi^{n} e^{i(m+2) k h}
\end{aligned}
$$

Now, if Euler's formula:

$$
e^{i k h}=\cos (k h)+i \sin (k h),
$$

is used in Eq. (20) and this equation is simplified, we get the following growth factor:

$$
\xi=\frac{a-i b}{a+i b},
$$

which gives:

$$
\begin{aligned}
& a=\gamma_{3}+\left(\gamma_{2}+\gamma_{4}\right) \cos (k h)+\left(\gamma_{1}+\gamma_{5}\right) \cos (2 k h), \\
& b=\left(\gamma_{4}-\gamma_{2}\right) \sin (k h)+\left(\gamma_{5}-\gamma_{1}\right) \sin (2 k h),
\end{aligned}
$$

where:

$$
\begin{aligned}
& \gamma_{1}=\left[1-E Z_{m}-M\right], \\
& \gamma_{2}=\left[26-10 E Z_{m}+2 M\right], \\
& \gamma_{3}=[66], \\
& \gamma_{4}=\left[26+10 E Z_{m}-2 M\right], \\
& \gamma_{5}=\left[1+E Z_{m}+M\right], \\
& m=0,1, \cdots, N, \\
& E=\frac{5}{2 h} \varepsilon \Delta t, \quad M=\frac{30}{h^{3}} \mu \Delta t .
\end{aligned}
$$

The modulus of $\xi$ is 1 and therefore, the linearized scheme is unconditionally stable.

\section{Error analysis}

The efficiency and acceptability of a numerical scheme depend on its stability and rate of convergence. In this study, we approximated the model problem using piecewise polynomials for spatial approximations with collocation approach and a method of lines for the temporal approximation. Here, we aim for a short discussion about the accuracy of the above-mentioned space time scheme without formal proof. For a detailed analysis and discussion about this issue, the readers can refer to Bochev and Gunzburger [39] and the references therein. Here, we use some constants $C_{i} \geq 0$, which are not necessarily the same in all the cases.

Polynomials play an important role in numerical integrations and analysis $[37,40]$. Global polynomial 
interpolations can be used to integrate the solutions to differential equations when the unknown curves are considered to be very smooth. However, there are many practical situations in engineering and physical problems that the solutions are not sufficiently smooth to support global polynomial approximation. In these cases, piecewise polynomial interpolations play an important role and work very well to integrate the solutions. One of the main benefits of using polynomial basis functions is that they have smooth curves. In general, if we have $k+1$ data points, then there is exactly one polynomial of degree at most $k$ passing through the data points and the error in the interpolating polynomial is proportional to the power of the distance between the data points. A detailed discussion about the polynomial approximation and least squares piecewise polynomials approximations can be found in $[37,39,40]$. Moreover, the main benefit of using collocation scheme is that it gives super-convergence pointwise approximation. Compared with the Galerkin inner product approach, the collocation approach does not require an extra integral for evaluation. Thus, this approach is simpler and more efficient to compute solutions.

Let $H^{r}(\Omega)$ be the space of $r$ times differentiable functions and $\|\cdot\|_{r}$ be the standard $H^{r}(\Omega)$ norm. Let $v_{h}$ be an approximation to a function $v(x) \in H^{r}(\Omega)$ in $\Omega$. Let $h$ be the distance between the grids and $\Omega=\cup_{i} \Omega_{i}$, where $\Omega_{i}=\left[x_{i}, x_{i+1}\right], x_{i+1}=x_{i}+h$. We observe $[37,40,41]$ that:

$$
\left\|v(x)-v_{h}(x)\right\| \leq C \Delta x^{k+1}\|v\|_{k+1}, \quad 1 \leq k<r,
$$

and $v_{h}$ stands for interpolation by piecewise polynomials of degree $r$ (considering $\Omega=\cup_{i} \Omega_{i}$ ). This error is preserved by the Galerkin finite element approximation as well [40].

It can be easily observed $[39,40]$ that if $w_{h}$ is a suitable B-spline defined by a polynomial of degree less than or equal to $k$, then:

$$
\left\|w(x)-w_{h}(x)\right\| \leq C \Delta x^{l+1}\|w\|_{l+1}, \quad 1 \leq l<k,
$$

for any $w \in H_{k}(\Omega)$. In this study, we use quintic Bsplines for space integration. Thus, from the above discussion, one sees that we obtain an $\mathcal{O}\left(\Delta x^{6}\right)$ accuracy for the spatial approximation in $L_{2}(\Omega)$ norm, because for time, we use the Crank-Nicolson scheme, which is of $\mathcal{O}\left(\Delta t^{2}\right)$ accuracy in $L_{2}([0, T])$ norm for some $T>0$, followed by a forward difference scheme, which is of $\mathcal{O}(\Delta t)$ accuracy in $L_{2}([0, T])$ norm for some $T>0$ [40]. Therefore, we obtain the error bound as:

$$
\begin{aligned}
\left\|u(x, t)-u_{h}(x, t)\right\| & \leq C_{1} \Delta x^{6}+C_{2} \Delta t^{2}+C_{3} \Delta t \\
& =C_{1} \Delta x^{6}+C_{2} \Delta t
\end{aligned}
$$

for suitable $C_{1} \geq 0$ and $C_{2} \geq 0$.

In addition, the convergence order of CrankNicolson method for the temporal variable is quadratic. The development of stability and higher order convergence scheme according to time variable is subject of the further investigations.

\section{Numerical simulations}

Numerical results of the gKdV equation are obtained for two problems: the motion of single solitary wave, evolution of solitons with Gaussian and undular bore initial conditions. We use the error norm $L_{2}$ that is defined as:

$$
L_{2}=\left\|U^{\text {exact }}-U_{N}\right\|_{2} \simeq \sqrt{h \sum_{j=1}^{N}\left|U_{j}^{\text {exact }}-\left(U_{N}\right)_{j}\right|^{2}},
$$

and the error norm $L_{\infty}$ :

$$
\begin{aligned}
L_{\infty} & =\left\|U^{\text {exact }}-U_{N}\right\|_{\infty} \simeq \max _{j}\left|U_{j}^{\text {exact }}-\left(U_{N}\right)_{j}\right| \\
j & =1,2, \cdots, N
\end{aligned}
$$

to calculate the difference between analytical and numerical solutions at some specified times. The generalized KdV equation (Eq. (2)) possesses only three invariants by:

$$
\begin{aligned}
I_{1} & =\int_{a}^{b} U d x \simeq h \sum_{j=1}^{N} U_{j}^{n}, \\
I_{2} & =\int_{a}^{b} U^{2} d x \simeq h \sum_{j=1}^{N}\left(U_{j}^{n}\right)^{2}, \\
I_{3} & =\int_{a}^{b}\left[U^{p+2}-\frac{\mu(p+1)(p+2)}{2 \varepsilon}\left(U_{x}\right)^{2}\right] d x \\
& \simeq h \sum_{j=1}^{N}\left[\left(U_{j}^{n}\right)^{p+2}-\frac{\mu(p+1)(p+2)}{2 \varepsilon}\left(U_{x}\right)_{j}^{2}\right],
\end{aligned}
$$

which correspond to conversation laws. In the simulation of solitary wave motion, the invariants $I_{1}, I_{2}$, and $I_{3}$ are monitored to check the conversation of the numerical algorithm.

\subsection{The motion of single solitary wave}

The single solitary wave solution of the gKdV equation (Eq. (2)) considered by the boundary conditions $U \rightarrow 0$ as $x \rightarrow \pm \infty$ is given by:

$$
U(x, t)=A \operatorname{sech}^{\frac{2}{p}}[k(x-c t)],
$$

where $A=\left[\frac{c(p+1)(p+2)}{2 \varepsilon}\right]^{\frac{1}{p}}$ and $k=\frac{p}{2} \sqrt{\frac{c}{\mu}}$ [36]. Note that, $c, \varepsilon, \mu$, and $p$ are arbitrary constants. The initial condition is: 


$$
U(x, 0)=A \operatorname{sech}^{\frac{2}{p}}(k x) .
$$

To show the motion of the single solitary wave solution numerically, let $\varepsilon=1, \mu=4.84 \times 10^{-4}, c=0.3$, and $x \epsilon[0,2]$. Now, we consider the following cases.

\subsubsection{Case $I$}

For $p=1$, parameters are chosen as $\varepsilon=1, \mu=4.84 \times$ $10^{-4}, c=0.3, h=0.01$, and $\Delta t=0.005$ to correspond with those in the earlier studies. For these parameters, the single solitary wave has the amplitude $=0.9$. The conserved quantities and error norms, $L_{2}$ and $L_{\infty}$, are shown at selected times up to time $t=3$. The obtained results are tabulated in Table 2 . It can be seen from Table 2 that the error norms $L_{2}$ and $L_{\infty}$ are small enough and the invariants are nearly unchanged during the process time. It is observed in the tables that percentages of relative changes of $I_{1}, I_{2}$, and $I_{3}$ are $6.33 \times 10^{-4}, 3.41 \times 10^{-7}$, and $7.53 \times 10^{-7}$, respectively. Table 2 represents a comparison of the values of the invariants and error norms obtained by the present method with earlier results. Numerical solution of single solitary wave is plotted at selected times from $t=0$ to $t=3$ in Figure 1 .

\subsubsection{Case II}

For $p=2$, parameters $\varepsilon=1, \mu=4.84 \times 10^{-4}$, and $c=0.3$ are considered to examine the quantities of the invariants and error norms for different space steps $(h)$ and time steps $(\Delta t)$. The single solitary wave has the amplitude $=1.34164$. The calculated values are presented in Table 3 . As can be seen in Table 3 , the error norms $L_{2}$ and $L_{\infty}$ are reasonably small and the invariants remain almost constant as the time increases. The agreement between numerical and analytical solutions is excellent. Table 3 shows a comparison of the values of the invariants and error

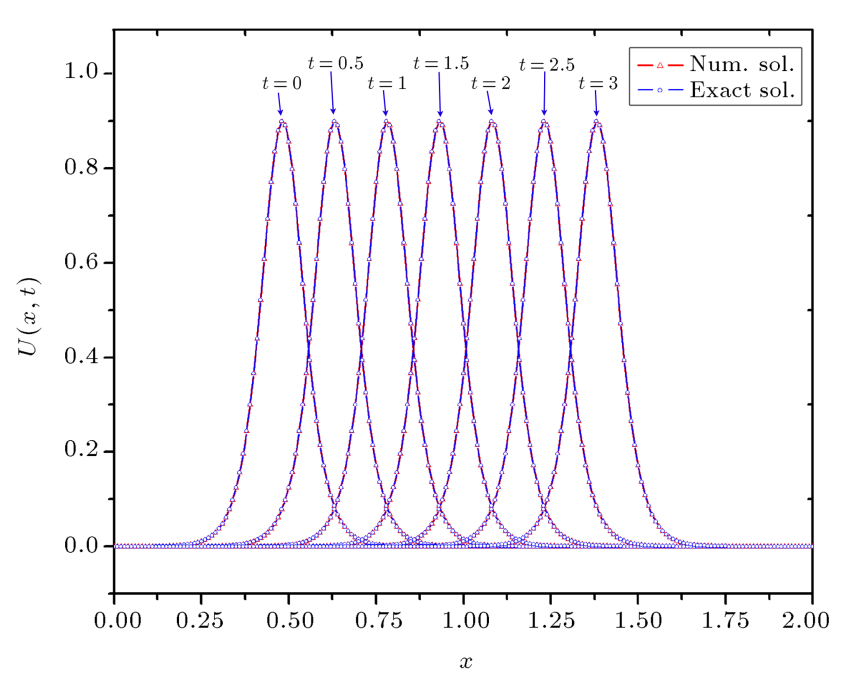

Figure 1. Motion of single solitary wave for $p=1, \varepsilon=1$, $\mu=4.84 \times 10^{-4}, c=0.3, h=0.01$, and $\Delta t=0.005$.

norms for different space and time steps. The motion of single solitary wave is depicted with $\varepsilon=1, \mu=$ $4.84 \times 10^{-4}, c=0.3, h=0.01$, and $\Delta t=0.005$ at selected times in Figure 2.

\subsubsection{Case III}

For $p=3$, parameters are taken to be $\varepsilon=1, \mu=$ $4.84 \times 10^{-4}$, and $c=0.3$ to analyze the quantities of the invariants and error norms for different space steps $(h)$ and time steps $(\Delta t)$. In this case, the single solitary wave has the amplitude $=1.44225$. The conserved quantities and error norms $L_{2}$ and $L_{\infty}$ are listed at selected times until the time $t=3$. As can be seen in Table 4 , the error norms $L_{2}$ and $L_{\infty}$ are sensibly small and the quantities of the invariants remain almost constant during the computer run. Table 4 displays a comparison of the values of the invariants and error

Table 2. Comparison of invariants and error norms for single solitary wave with $p=1, \varepsilon=1, \mu=4.84 \times 10^{-4}, c=0.3$, $h=0.01$, and $\Delta t=0.005$ at $0 \leq x \leq 2$.

\begin{tabular}{|c|c|c|c|c|c|c|}
\hline$t$ & & $I_{1}$ & $I_{2}$ & $I_{3}$ & $L_{2} \times 10^{3}$ & $L_{\infty} \times 10^{3}$ \\
\hline 0.0 & & 0.144598 & 0.086759 & 0.046850 & 0.000000 & 0.000000 \\
\hline 0.5 & & 0.144601 & 0.086759 & 0.046850 & 0.044089 & 0.127200 \\
\hline 1.0 & & 0.144599 & 0.086759 & 0.046850 & 0.079487 & 0.238623 \\
\hline 1.5 & & 0.144599 & 0.086759 & 0.046850 & 0.114742 & 0.324535 \\
\hline 2.0 & & 0.144600 & 0.086759 & 0.046850 & 0.151352 & 0.419257 \\
\hline 2.5 & & 0.144599 & 0.086759 & 0.046850 & 0.187263 & 0.500722 \\
\hline 3.0 & & 0.144599 & 0.086759 & 0.046850 & 0.227130 & 0.619010 \\
\hline 3.0 & [30] & 0.144597 & 0.086761 & 0.046852 & 0.038684 & - \\
\hline 3.0 & [31] & 0.144601 & 0.086760 & 0.046850 & - & - \\
\hline 3.0 & {$[32]$} & 0.144600 & 0.086759 & 0.046850 & 0.387274 & 1.041563 \\
\hline 3.0 & [33] & 0.144597 & 0.086759 & 0.046849 & - & 1.61 \\
\hline 3.0 & [34] & 0.14460 & 0.086761 & 0.046876 & - & - \\
\hline 3.0 & {$[35]$} & 0.14460 & 0.08676 & 0.04685 & - & - \\
\hline
\end{tabular}


Table 3. Comparison of invariants and error norms for single solitary wave with $p=2, \varepsilon=1, \mu=4.84 \times 10^{-4}$, and $c=0.3$ at $0 \leq x \leq 2$.

\begin{tabular}{|c|c|c|c|c|c|c|}
\hline & $t$ & $L_{2} \times 10^{3}$ & $L_{\infty} \times 10^{3}$ & $I_{1}$ & $I_{2}$ & $I_{3}$ \\
\hline \multirow{7}{*}{$\begin{aligned} h & =0.01 \\
\Delta t & =0.005\end{aligned}$} & 0.0 & 0.000000 & 0.000000 & 0.169296 & 0.144599 & 0.086760 \\
\hline & 0.5 & 0.161944 & 0.574891 & 0.169300 & 0.144599 & 0.086760 \\
\hline & 1.0 & 0.318711 & 1.054684 & 0.169292 & 0.144599 & 0.086760 \\
\hline & 1.5 & 0.460453 & 1.582264 & 0.169294 & 0.144599 & 0.086760 \\
\hline & 2.0 & 0.607800 & 1.948279 & 0.169295 & 0.144599 & 0.086760 \\
\hline & 2.5 & 0.752947 & 2.376394 & 0.169297 & 0.144599 & 0.086760 \\
\hline & 3.0 & 0.912455 & 2.886583 & 0.169297 & 0.144599 & 0.086760 \\
\hline \multirow{7}{*}{$\begin{aligned} h & =0.005 \\
\Delta t & =0.0025\end{aligned}$} & 0.0 & 0.000000 & 0.000000 & 0.169296 & 0.144599 & 0.086759 \\
\hline & 0.5 & 0.063939 & 0.208079 & 0.169298 & 0.144599 & 0.086759 \\
\hline & 1.0 & 0.125994 & 0.397920 & 0.169297 & 0.144599 & 0.086759 \\
\hline & 1.5 & 0.188595 & 0.585358 & 0.169297 & 0.144599 & 0.086759 \\
\hline & 2.0 & 0.250310 & 0.769922 & 0.169297 & 0.144599 & 0.086759 \\
\hline & 2.5 & 0.313310 & 0.977050 & 0.169297 & 0.144599 & 0.086759 \\
\hline & 3.0 & 0.375889 & 1.158590 & 0.169297 & 0.144599 & 0.086759 \\
\hline \multirow{7}{*}{$\begin{aligned} h & =0.001 \\
\Delta t & =0.0005\end{aligned}$} & 0.0 & 0.000000 & 0.000000 & 0.169298 & 0.144601 & 0.086763 \\
\hline & 0.5 & 0.012709 & 0.034070 & 0.169300 & 0.144601 & 0.086763 \\
\hline & 1.0 & 0.020823 & 0.056822 & 0.169299 & 0.144601 & 0.086763 \\
\hline & 1.5 & 0.023853 & 0.069660 & 0.169299 & 0.144601 & 0.086763 \\
\hline & 2.0 & 0.058242 & 0.209984 & 0.169299 & 0.144601 & 0.086763 \\
\hline & 2.5 & 0.113519 & 0.380739 & 0.169299 & 0.144601 & 0.086763 \\
\hline & 3.0 & 0.168194 & 0.543238 & 0.169300 & 0.144601 & 0.086763 \\
\hline
\end{tabular}

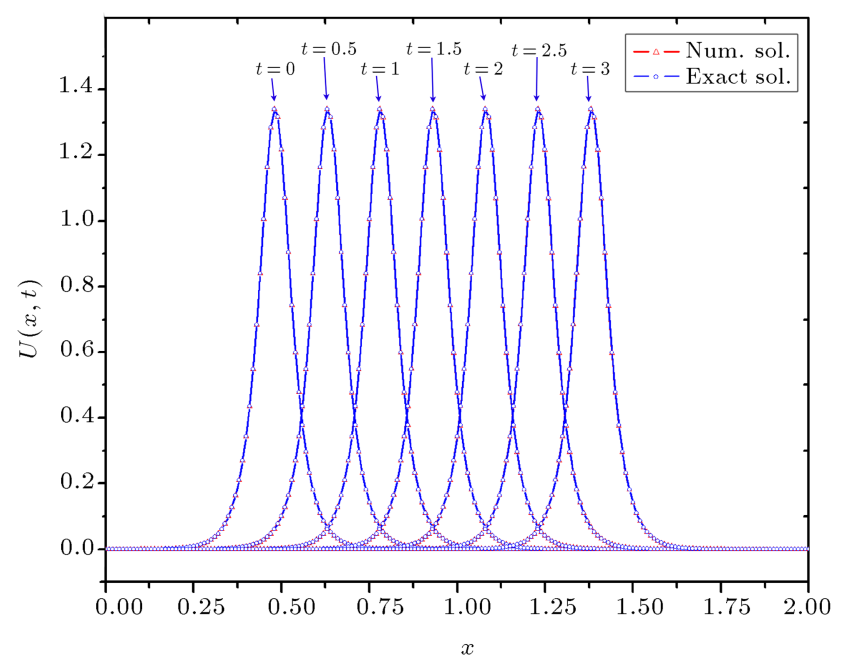

Figure 2. Motion of single solitary wave for $p=2, \varepsilon=1$, $\mu=4.84 \times 10^{-4}, c=0.3, h=0.01$, and $\Delta t=0.005$.

norms for different space and time steps. In Figure 3, the propagation of single solitary wave is illustrated with $\varepsilon=1, \mu=4.84 \times 10^{-4}, c=0.3, h=0.01$, and $\Delta t=0.005$ at selected times from $t=0$ to $t=3$.

Finally, for $p=1,2$, and 3 , errors distributions at time $t=3$ are depicted in Figure 4 to show the errors

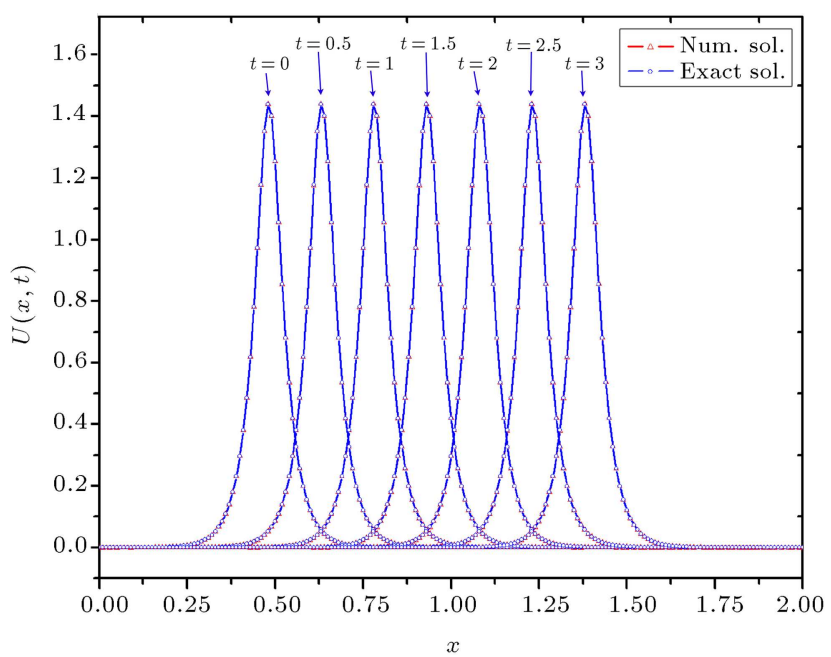

Figure 3. Motion of single solitary wave for $p=3, \varepsilon=1$, $\mu=4.84 \times 10^{-4}, c=0.3, h=0.01$, and $\Delta t=0.005$.

between the analytical and numerical results over the problem domain. Also, the invariants and error norms are examined in Table 5 for different values of $p$.

In the light of all these studies, it is observed that as the value of $p$ increases, amplitude of the single solitary waves, error norm, and relative changes of the conserved quantities increase. 
Table 4. Comparison of invariants and error norms for single solitary wave with $p=3, \varepsilon=1, \mu=4.84 \times 10^{-4}$, and $c=0.3$ at $0 \leq x \leq 2$

\begin{tabular}{|c|c|c|c|c|c|c|}
\hline & $t$ & $L_{2} \times 10^{3}$ & $L_{\infty} \times 10^{3}$ & $I_{1}$ & $I_{2}$ & $I_{3}$ \\
\hline \multirow{7}{*}{$\begin{aligned} h & =0.01 \\
\Delta t & =0.005\end{aligned}$} & 0.0 & 0.000000 & 0.000000 & 0.162456 & 0.144101 & 0.061761 \\
\hline & 0.5 & 0.178756 & 0.692042 & 0.162463 & 0.144100 & 0.061758 \\
\hline & 1.0 & 0.234132 & 0.744727 & 0.162445 & 0.144100 & 0.061757 \\
\hline & 1.5 & 0.470967 & 1.328716 & 0.162442 & 0.144100 & 0.061756 \\
\hline & 2.0 & 0.502231 & 1.490645 & 0.162452 & 0.144099 & 0.061754 \\
\hline & 2.5 & 0.540347 & 1.721624 & 0.162451 & 0.144099 & 0.061752 \\
\hline & 3.0 & 0.554489 & 1.937605 & 0.162460 & 0.144097 & 0.061749 \\
\hline \multirow{7}{*}{$\begin{aligned} h & =0.005 \\
\Delta t & =0.0025\end{aligned}$} & 0.0 & 0.000000 & 0.000000 & 0.162456 & 0.144101 & 0.061758 \\
\hline & 0.5 & 0.174879 & 0.613433 & 0.162458 & 0.144101 & 0.061758 \\
\hline & 1.0 & 0.367348 & 1.257014 & 0.162458 & 0.144101 & 0.061758 \\
\hline & 1.5 & 0.567146 & 1.912527 & 0.162458 & 0.144101 & 0.061758 \\
\hline & 2.0 & 0.769779 & 2.589902 & 0.162458 & 0.144101 & 0.061758 \\
\hline & 2.5 & 0.973379 & 3.255744 & 0.162456 & 0.144101 & 0.061758 \\
\hline & 3.0 & 1.180900 & 3.939972 & 0.162456 & 0.144101 & 0.061758 \\
\hline \multirow{7}{*}{$\begin{aligned} h & =0.001 \\
\Delta t & =0.0005\end{aligned}$} & 0.0 & 0.000000 & 0.000000 & 0.162458 & 0.144103 & 0.061763 \\
\hline & 0.5 & 0.032541 & 0.107203 & 0.162460 & 0.144103 & 0.061763 \\
\hline & 1.0 & 0.073624 & 0.248928 & 0.162458 & 0.144103 & 0.061763 \\
\hline & 1.5 & 0.116540 & 0.388806 & 0.162458 & 0.144103 & 0.061763 \\
\hline & 2.0 & 0.192098 & 0.667439 & 0.162458 & 0.144103 & 0.061763 \\
\hline & 2.5 & 0.292241 & 1.003527 & 0.162459 & 0.144103 & 0.061763 \\
\hline & 3.0 & 0.396268 & 1.348702 & 0.162460 & 0.144103 & 0.061763 \\
\hline
\end{tabular}
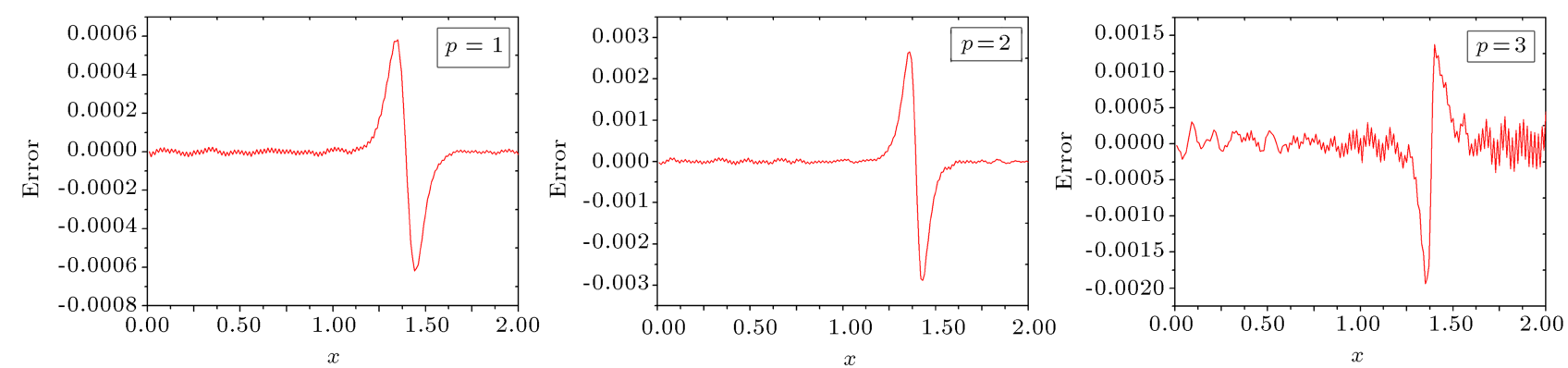

Figure 4. Errors for $\varepsilon=1, \mu=4.84 \times 10^{-4}, c=0.3, h=0.01, \Delta t=0.005$, and different values of $p$ at $t=12.5$.

\subsection{Evolution of solitons}

\subsubsection{Gaussian initial condition}

Evolution of a train of solitons of the gKdV equation is studied using the Gaussian initial condition:

$$
U(x, 0)=\exp \left(-x^{2}\right)
$$

and boundary condition:

$$
U(-15, t)=U(15, t)=0, \quad t>0,
$$

for various values of $\mu$. In this case, the behavior of the solution depends on the value of $\mu$. The critical value for the Gaussian initial condition used in some simulations is $\mu_{c}=0.0625[29,42]$. For $\mu \ll \mu_{c}$, the initial Gaussian breaks up into a number of solitons, while the actual number depends on the size of $\mu$. For $\mu \gg \mu_{c}$, the Gaussian condition does not break up into solitons, but exhibits rapidly oscillating wave packets. Therefore, the values of $\mu=0.04, \mu=0.01$, and $\mu=$ 0.001 are chosen for different values of space step $(h)$ and time step $(\Delta t)$ at $0 \leqslant t \leqslant 12.5$.

Numerical computations are done for different values of $p$. Nonlinearity effect is investigated in the following three cases:

Case I. For $p=1$, parameter $\varepsilon=1$ is chosen. The numerical computations are done up to $t=12.5$. The values of the three invariants of motion for various 
Table 5. Invariants and error norms for single solitary wave with $\varepsilon=1, \mu=4.84 \times 10^{-4}, c=0.3, h=0.01$, and $\Delta t=0.005$.

\begin{tabular}{|c|c|c|c|c|c|c|}
\hline & $t$ & $L_{2} \times 10^{3}$ & $L_{\infty} \times 10^{3}$ & $I_{1}$ & $I_{2}$ & $I_{3}$ \\
\hline \multirow{7}{*}{$p=1$} & 0.0 & 0.000000 & 0.000000 & 0.144598 & 0.086759 & 0.046850 \\
\hline & 0.5 & 0.044089 & 0.127200 & 0.144601 & 0.086759 & 0.046850 \\
\hline & 1.0 & 0.079487 & 0.238623 & 0.144599 & 0.086759 & 0.046850 \\
\hline & 1.5 & 0.114742 & 0.324535 & 0.144599 & 0.086759 & 0.046850 \\
\hline & 2.0 & 0.151352 & 0.419257 & 0.144600 & 0.086759 & 0.046850 \\
\hline & 2.5 & 0.187263 & 0.500722 & 0.144599 & 0.086759 & 0.046850 \\
\hline & 3.0 & 0.227130 & 0.619010 & 0.144599 & 0.086759 & 0.046850 \\
\hline \multirow{7}{*}{$p=2$} & 0.0 & 0.000000 & 0.000000 & 0.169296 & 0.144599 & 0.086760 \\
\hline & 0.5 & 0.161944 & 0.574891 & 0.169300 & 0.144599 & 0.086760 \\
\hline & 1.0 & 0.318711 & 1.054684 & 0.169292 & 0.144599 & 0.086760 \\
\hline & 1.5 & 0.460453 & 1.582264 & 0.169294 & 0.144599 & 0.086760 \\
\hline & 2.0 & 0.607800 & 1.948279 & 0.169295 & 0.144599 & 0.086760 \\
\hline & 2.5 & 0.752947 & 2.376394 & 0.169297 & 0.144599 & 0.086760 \\
\hline & 3.0 & 0.912455 & 2.886583 & 0.169297 & 0.144599 & 0.086760 \\
\hline \multirow{7}{*}{$p=3$} & 0.0 & 0.000000 & 0.000000 & 0.162456 & 0.144101 & 0.061761 \\
\hline & 0.5 & 0.178756 & 0.692042 & 0.162463 & 0.144100 & 0.061758 \\
\hline & 1.0 & 0.234132 & 0.744727 & 0.162445 & 0.144100 & 0.061757 \\
\hline & 1.5 & 0.470967 & 1.328716 & 0.162442 & 0.144100 & 0.061756 \\
\hline & 2.0 & 0.502231 & 1.490645 & 0.162452 & 0.144099 & 0.061754 \\
\hline & 2.5 & 0.540347 & 1.721624 & 0.162451 & 0.144099 & 0.061752 \\
\hline & 3.0 & 0.554489 & 1.937605 & 0.162460 & 0.144097 & 0.061749 \\
\hline
\end{tabular}

Table 6. Invariants for Gaussian initial condition with $p=1, \varepsilon=1$, and $c=0.3$ at $-15 \leq x \leq 15$.

\begin{tabular}{|c|c|c|c|c|c|c|c|c|c|}
\hline \multirow{2}{*}{$\begin{array}{c}p=1 \\
t\end{array}$} & \multicolumn{3}{|c|}{$\mu=0.04$} & \multicolumn{3}{|c|}{$\boldsymbol{\mu}=\mathbf{0 . 0 1}$} & \multicolumn{3}{|c|}{$\mu=0.001$} \\
\hline & $I_{1}$ & $I_{2}$ & $I_{3}$ & $I_{1}$ & $I_{2}$ & $I_{3}$ & $I_{1}$ & $I_{2}$ & $I_{3}$ \\
\hline 0.0 & 1.772454 & 1.253314 & 0.872929 & 1.772454 & 1.253314 & 0.985727 & 1.772454 & 1.253314 & 1.019567 \\
\hline 2.5 & 1.772484 & 1.253314 & 0.872921 & 1.772458 & 1.253324 & 0.985589 & 1.772452 & 1.253317 & 1.019561 \\
\hline 5.0 & 1.772454 & 1.253315 & 0.872919 & 1.772419 & 1.253344 & 0.985538 & 1.772454 & 1.253319 & 1.019547 \\
\hline 7.5 & 1.774643 & 1.253828 & 0.872915 & 1.772553 & 1.253363 & 0.985557 & 1.772458 & 1.253320 & 1.019547 \\
\hline 10.0 & 1.774464 & 1.253579 & 0.872979 & 1.772360 & 1.253381 & 0.985579 & 1.772447 & 1.253321 & 1.019548 \\
\hline \multirow[t]{2}{*}{12.5} & 1.763884 & 1.257298 & 0.872872 & 1.772730 & 1.253429 & 0.985626 & 1.772452 & 1.253321 & 1.019549 \\
\hline & $h=0.1$ & & $\Delta t=0.01$ & $h=0.1$ & & $\Delta t=0.01$ & $h=0.01$ & & $\Delta t=0.005$ \\
\hline
\end{tabular}

values of $\mu$, space step, and time step are presented in Table 6. Also, Figure 5 illustrates the development of the Gaussian initial condition into solitons at $t=$ 12.5. As it is seen from Figure 5, a soliton and an oscillating tail are drawn for $\mu=0.04$. Three solitons are found when $\mu=0.01$. Nine solitons moving to the right are observed for $\mu=0.001$.

Case II. For $p=2$, computations are performed with $\varepsilon=1$ carried out from $t=0$ to $t=12.5$. In
Table 7 , the values of the three invariants of motion for different values of $\mu$, space step, and time step are tabulated. For $\mu=0.04$, a soliton and an oscillating tail are visualized. When $\mu=0.01$, two solitons are shown. For $\mu=0.001$, six solitons moving to the right are illustrated. These cases are shown in Figure 6 at $t=12.5$.

Case III. For $p=3$, computations are accomplished by taking the parameter $\varepsilon=1$ from $t=0$ to 

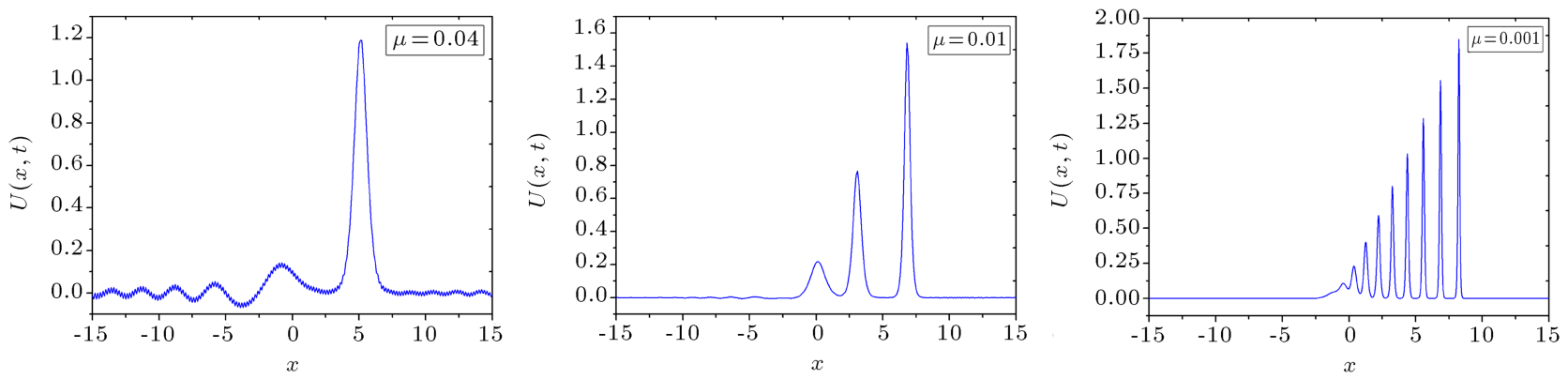

Figure 5. Generated waves for $p=1, \varepsilon=1$, and $c=0.3$ at $t=12.5$.
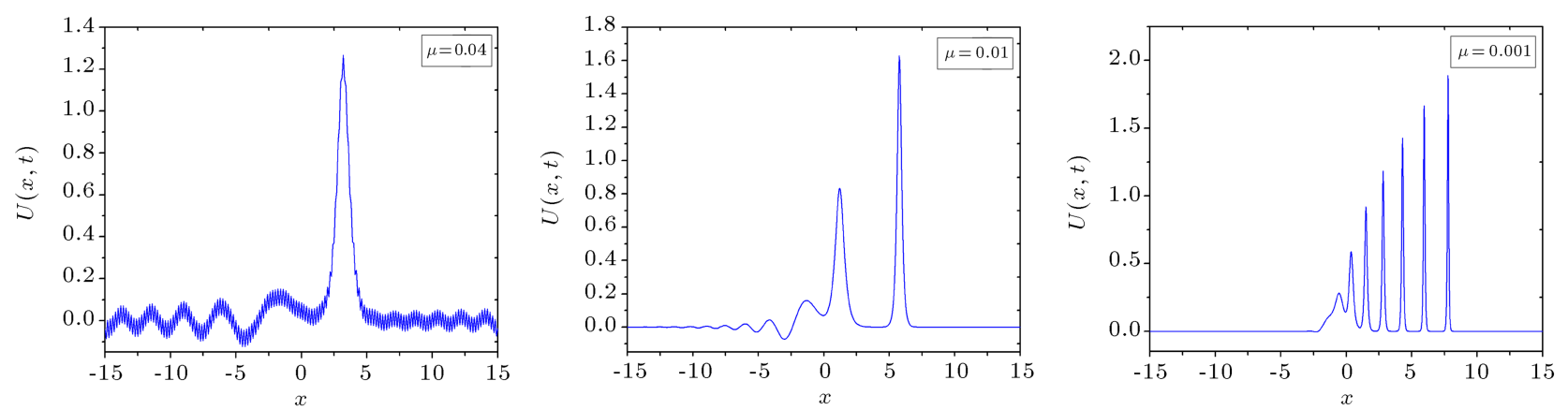

Figure 6. Generated waves for $p=2, \varepsilon=1$, and $c=0.3$ at $t=12.5$.

Table 7. Invariants for Gaussian initial condition with $p=2, \varepsilon=1$, and $c=0.3$ at $-15 \leq x \leq 15$.

\begin{tabular}{|c|c|c|c|c|c|c|c|c|c|}
\hline \multirow{2}{*}{$\begin{array}{c}p=2 \\
t\end{array}$} & \multicolumn{3}{|c|}{$\mu=0.04$} & \multicolumn{3}{|c|}{$\mu=0.01$} & \multicolumn{3}{|c|}{$\mu=0.001$} \\
\hline & $I_{1}$ & $I_{2}$ & $I_{3}$ & $I_{1}$ & $I_{2}$ & $I_{3}$ & $I_{1}$ & $I_{2}$ & $I_{3}$ \\
\hline 0.0 & 1.772454 & 1.253314 & 0.585431 & 1.772454 & 1.253314 & 0.811028 & 1.772455 & 1.253315 & 0.878707 \\
\hline 2.5 & 1.772509 & 1.253315 & 0.585402 & 1.772453 & 1.253315 & 0.810976 & 1.772455 & 1.253314 & 0.878859 \\
\hline 5.0 & 1.770902 & 1.254005 & 0.585309 & 1.772449 & 1.253315 & 0.810930 & 1.772453 & 1.253309 & 0.878865 \\
\hline 7.5 & 1.782043 & 1.263829 & 0.583137 & 1.772450 & 1.253316 & 0.810927 & 1.772451 & 1.253303 & 0.878846 \\
\hline 10.0 & 1.784649 & 1.263990 & 0.585781 & 1.772460 & 1.253321 & 0.810929 & 1.772449 & 1.253298 & 0.878826 \\
\hline \multirow[t]{2}{*}{12.5} & 1.743934 & 1.300404 & 0.573455 & 1.772538 & 1.253318 & 0.810930 & 1.772447 & 1.253292 & 0.878806 \\
\hline & $h=0.1$ & & $\Delta t=0.01$ & $h=0.05$ & & $\Delta t=0.01$ & $h=0.01$ & & $\Delta t=0.005$ \\
\hline
\end{tabular}

$t=12.5$. The values of the three invariants of motion for various values of $\mu$, space step, and time step are given in Table 8 . For $\mu=0.04$, a soliton and an oscillating tail are formed. When $\mu=0.01$, two solitons are shown. For $\mu=0.001$, five solitons moving to the right are drawn. Also, Figure 7 displays the evolution of the solitons at $t=12.5$.

Consequently, the initial perturbation breaks up into a number of solitons in the course of time depending on the chosen value of $\mu$. Thus, if the value of $\mu$ is decreased, then the number of solitons, amplitude, and velocity increase with the same value of $p$. Also, as $p$ increases, the number of solitons decreases at selected times.

\subsubsection{Undular bore initial condition}

As the last test problem, evolution of a train of solitons of the gKdV equation are studied using the undular bore initial condition:

$$
U(x, 0)=\frac{1}{2} U_{0}\left[1-\tanh \left(\frac{|x|-x_{0}}{d}\right)\right],
$$

and boundary condition:

$$
U(-50, t)=U(150, t)=0, \quad t>0,
$$

leading to the production of a train of solitons depending upon the value $\mu$ of $g K d V$ equation. The 
Table 8. Invariants for Gaussian initial condition with $p=3, \varepsilon=1$, and $c=0.3$ at $-15 \leq x \leq 15$.

\begin{tabular}{|c|c|c|c|c|c|c|c|c|c|}
\hline \multirow{2}{*}{$\begin{array}{c}p=3 \\
t\end{array}$} & \multicolumn{3}{|c|}{$\boldsymbol{\mu}=0.04$} & \multicolumn{3}{|c|}{$\mu=0.01$} & \multicolumn{3}{|c|}{$\mu=0.001$} \\
\hline & $I_{1}$ & $I_{2}$ & $I_{3}$ & $I_{1}$ & $I_{2}$ & $I_{3}$ & $I_{1}$ & $I_{2}$ & $I_{3}$ \\
\hline 0.0 & 1.772454 & 1.253314 & 0.291340 & 1.772454 & 1.253314 & 0.667334 & 1.772455 & 1.253315 & 0.780133 \\
\hline 2.5 & 1.772324 & 1.253317 & 0.291287 & 1.772455 & 1.253314 & 0.667327 & 1.770654 & 1.247983 & 0.740775 \\
\hline 5.0 & 1.769279 & 1.256297 & 0.290132 & 1.772449 & 1.253314 & 0.667303 & 1.766790 & 1.236789 & 0.663718 \\
\hline 7.5 & 1.786485 & 1.276797 & 0.279958 & 1.772606 & 1.253325 & 0.667292 & 1.765355 & 1.232777 & 0.639189 \\
\hline 10.0 & 1.791897 & 1.281378 & 0.287956 & 1.772004 & 1.253400 & 0.667229 & 1.764482 & 1.230370 & 0.625128 \\
\hline \multirow[t]{2}{*}{12.5} & 1.736617 & 1.326327 & 0.249125 & 1.772970 & 1.253375 & 0.667288 & 1.763848 & 1.228627 & 0.615232 \\
\hline & $h=0.1$ & & $\Delta t=0.01$ & $h=0.025$ & & $\Delta t=0.005$ & $h=0.01$ & & $\Delta t=0.005$ \\
\hline
\end{tabular}
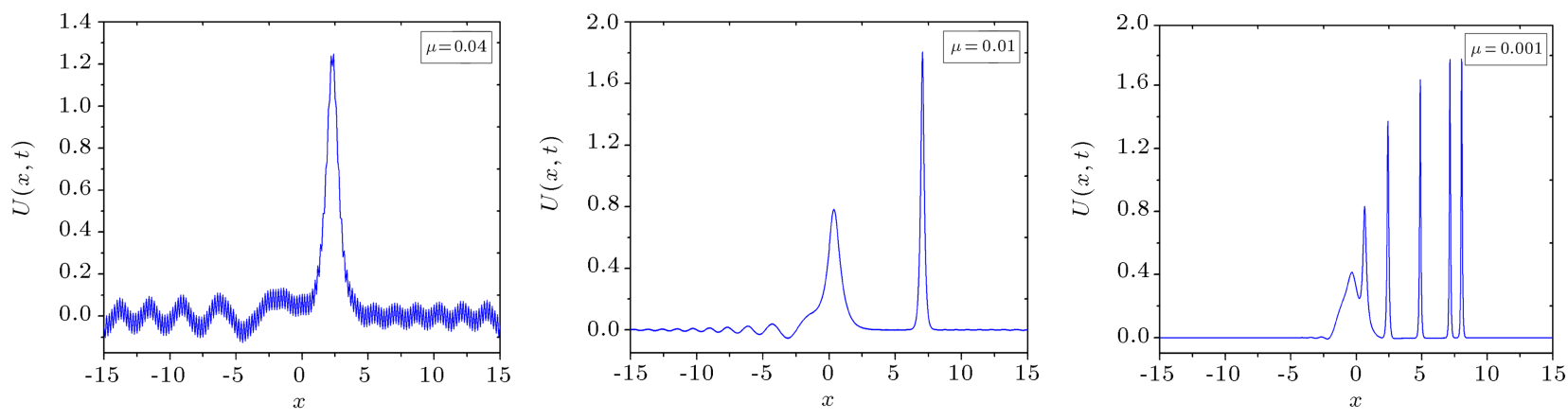

Figure 7. Generated waves for $p=3, \varepsilon=1$, and $c=0.3$ at $t=12.5$.

undular bore reflects the elevation of the water above the equilibrium surface at time $t=0$. The change in water level of magnitude is centered on $x=x_{0}$ and $d$ measures the steepness of the change. The smaller the value of $d$, the steeper is the slope. To be consistent with the papers [27-30], the parameters $\varepsilon=0.2, \mu=0.1, U_{0}=1, x_{0}=25$, and $d=5$ are considered for different values of $p$.

Case I. For $p=1$, parameters are taken to be $\varepsilon=0.2, \mu=0.1, c=0.3, h=0.4$, and $\Delta t=0.05$. The program is run up to time $t=800$. The three computed invariants are presented in Table 9. Figure 8 shows that the initial perturbation evolves into a well-developed train of solitons.

Case II. For $p=2$, parameters are chosen as $\varepsilon=0.2$, $\mu=0.1, c=0.3, h=0.4$, and $\Delta t=0.05$. Simulation is done up to time $t=800$. In Table 10, the values of the three invariants of motion are reported. It is seen from Figure 9 that as the simulation proceeds, undulations begin to develop and grow.

Case III. For $p=3$, parameters are taken as $\varepsilon=$ $0.2, \mu=0.1, c=0.3, h=0.1$, and $\Delta t=0.05$. The program proceeds until time $t=800$. The obtained values of the three invariants are tabulated in Table 11. The developed undular bore is simulated in Figure 10 at selected times. It is observed from the Figure 10 that train of solitons disintegrates at $t=800$.

As a result, as the value of $p$ increases, train of the solitons breaks down. In addition, relative changes of

Table 9. Invariants for undular bore initial condition with $p=1, \varepsilon=0.2, \mu=0.1, c=0.3, h=0.4$, and $\Delta t=0.05$ at $-50 \leq x \leq 150$.

\begin{tabular}{cccc}
\hline $\boldsymbol{t}$ & $\boldsymbol{I}_{\mathbf{1}}$ & $\boldsymbol{I}_{\mathbf{2}}$ & $\boldsymbol{I}_{\mathbf{3}}$ \\
\hline 0.0 & 50.000150 & 45.000479 & 42.300704 \\
100 & 50.000975 & 45.000496 & 42.300465 \\
200 & 49.995691 & 45.000979 & 42.299923 \\
300 & 50.013786 & 45.002598 & 42.300566 \\
400 & 50.020459 & 45.004304 & 42.301412 \\
500 & 50.027740 & 45.010674 & 42.305288 \\
600 & 50.009887 & 45.003685 & 42.300962 \\
700 & 49.972813 & 45.002301 & 42.300189 \\
800 & 49.937274 & 45.018632 & 42.310068 \\
\hline
\end{tabular}



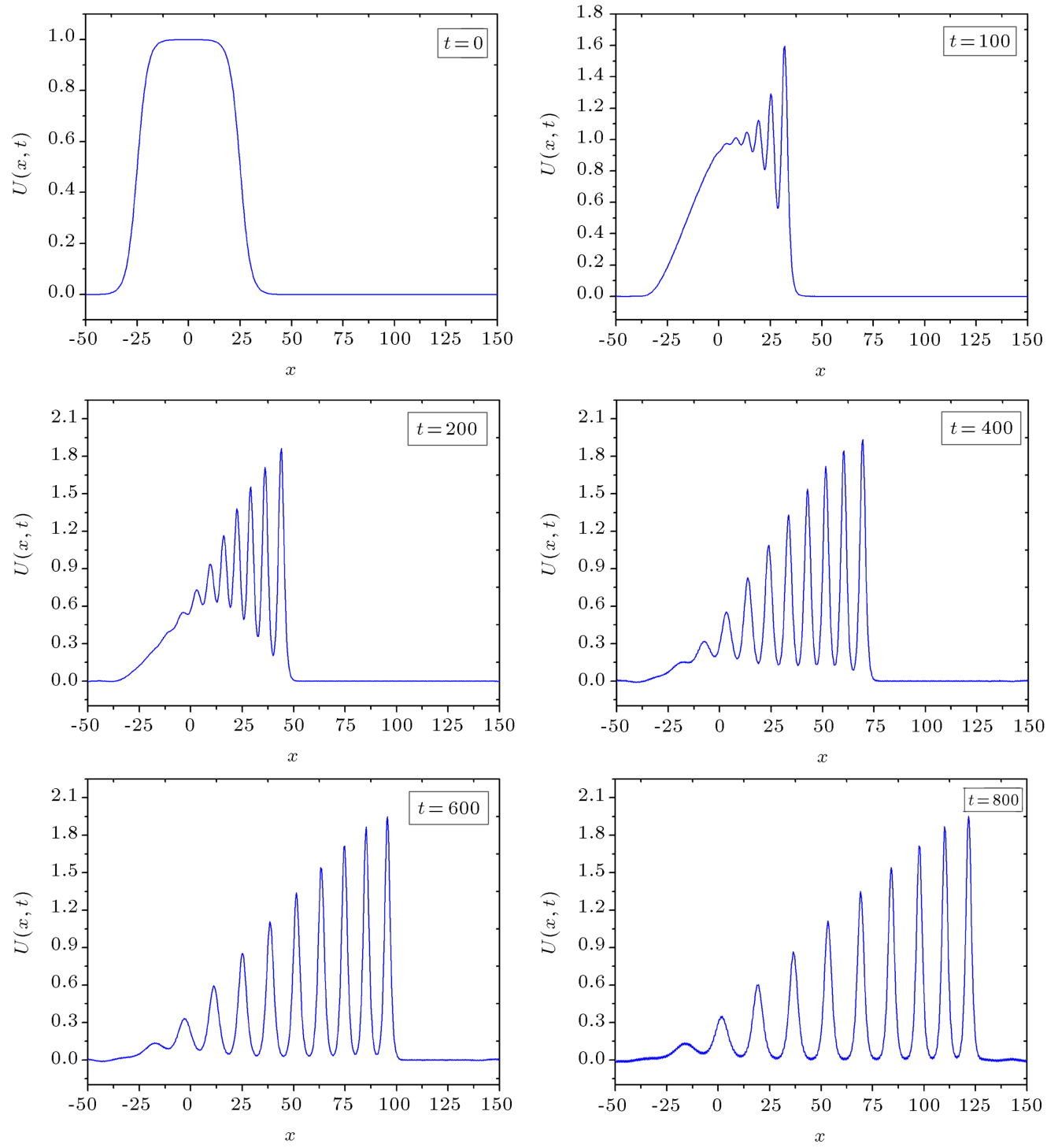

Figure 8. Developed train of solitons with $p=1, \varepsilon=0.2, \mu=0.1, c=0.3, h=0.4$, and $\Delta t=0.05$ at selected times.

Table 10. Invariants for undular bore initial condition with $p=2, \varepsilon=0.2, \mu=0.1, c=0.3, h=0.4$, and $\Delta t=0.05$ at $-50 \leq x \leq 150$.

\begin{tabular}{cccc}
\hline $\boldsymbol{t}$ & $\boldsymbol{I}_{\mathbf{1}}$ & $\boldsymbol{I}_{\mathbf{2}}$ & $\boldsymbol{I}_{\boldsymbol{3}}$ \\
\hline 0.0 & 50.000150 & 45.000479 & 40.434264 \\
100 & 50.002451 & 45.000654 & 40.431994 \\
200 & 49.963562 & 45.028383 & 40.452606 \\
300 & 50.033638 & 45.011934 & 40.445650 \\
400 & 50.126260 & 45.207891 & 40.703052 \\
500 & 50.093727 & 45.101218 & 40.576255 \\
600 & 50.096516 & 45.168962 & 40.782710 \\
700 & 50.072840 & 45.207062 & 40.759703 \\
800 & 49.978259 & 45.064224 & 40.577790 \\
\hline
\end{tabular}

Table 11. Invariants for undular bore initial condition with $p=3, \varepsilon=0.2, \mu=0.1, c=0.3, h=0.1$, and $\Delta t=0.05$ at $-50 \leq x \leq 150$.

\begin{tabular}{cccc}
\hline $\boldsymbol{t}$ & $\boldsymbol{I}_{\mathbf{1}}$ & $\boldsymbol{I}_{\mathbf{2}}$ & $\boldsymbol{I}_{\mathbf{3}}$ \\
\hline 0.0 & 50.000003 & 45.000355 & 38.917721 \\
100 & 50.001965 & 45.000375 & 38.917742 \\
200 & 49.952482 & 45.052395 & 38.950250 \\
300 & 50.043566 & 45.018658 & 38.921126 \\
400 & 50.153590 & 45.239066 & 39.122895 \\
500 & 50.268269 & 46.035793 & 39.887225 \\
600 & 50.138874 & 45.333503 & 39.183510 \\
700 & 49.932806 & 44.998598 & 38.852286 \\
800 & 45.104351 & 45.258168 & -69.395007 \\
\hline
\end{tabular}



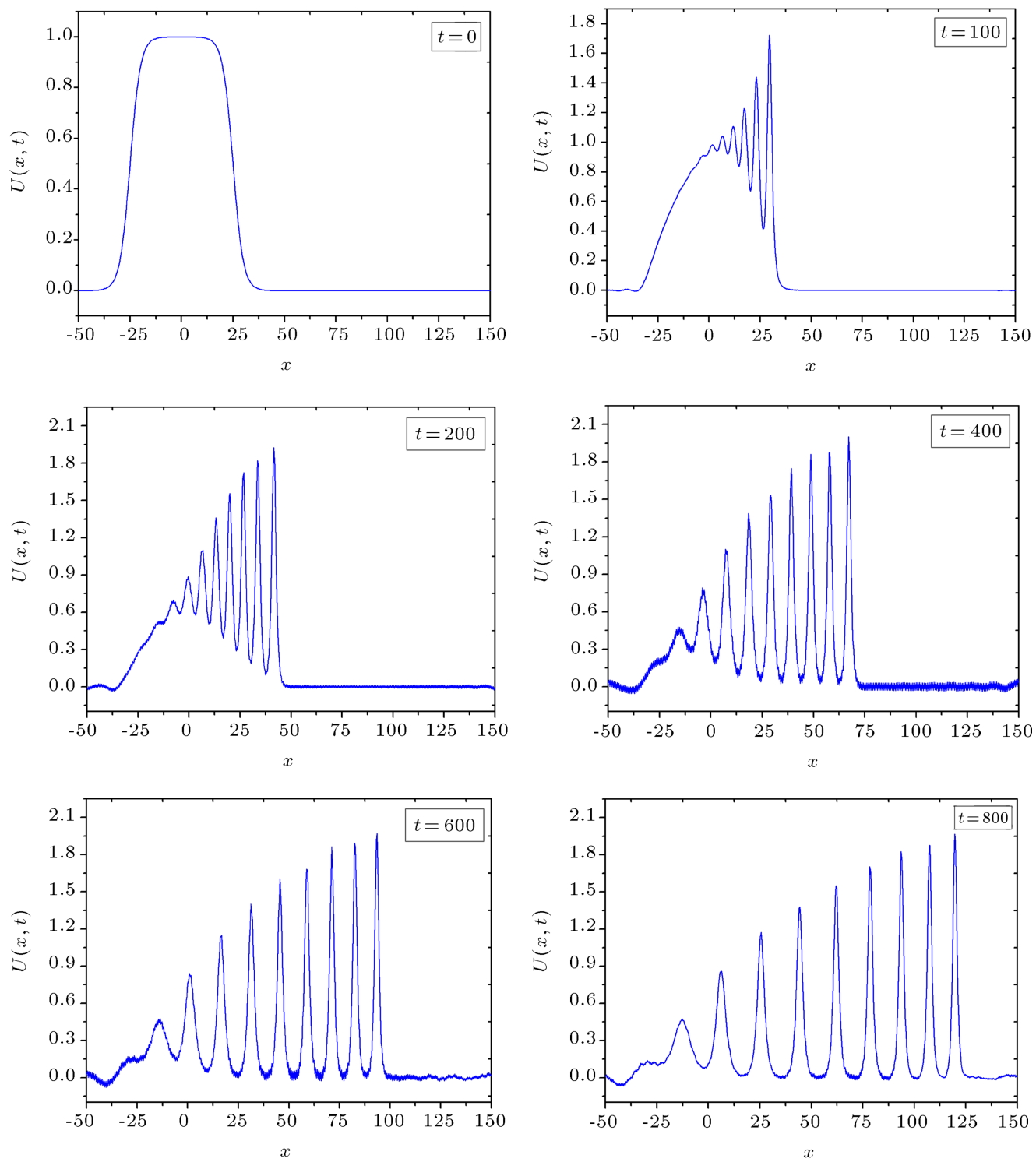

Figure 9. Developed train of solitons with $p=2, \varepsilon=0.2, \mu=0.1, c=0.3, h=0.4$, and $\Delta t=0.05$ at selected times.

the conserved quantities increase and the number of solitons decreases.

\section{Conclusion}

This paper studied $\mathrm{KdV}$ equation with power law nonlinearity to understand the dynamics of shallow water waves. The numerical scheme implemented in this paper was collocation finite element approach. The results gave way to meaningful simulations that were of great value in this study. A few special cases for power law nonlinearity were considered to illustrate the scheme. It was shown that $\mathrm{KdV}$ equation with power law nonlinearity failed to maintain stability for a particular value of this parameter.

The results of this paper stand on a strong footing for further research in future. The perturbation terms need to be included for a complete study of shallow water waves. These perturbations are inevitable and, thus, cannot be ignored. Two layered shallow water waves need to be considered as well. In this context, Bona-Chen model and Gear-Grimshaw equations can be studied. This will lead to several interesting and important observations that will be of prime importance in shallow water wave dynamics. This numerical scheme will later be applied to other models as enumerated earlier in this paper. The new results in this direction will be a project of our future work and will be communicated in due course.

\section{Acknowledgment}

The seventh author (Anjan Biswas) would like to thank Tshwane University of Technology for the academic 

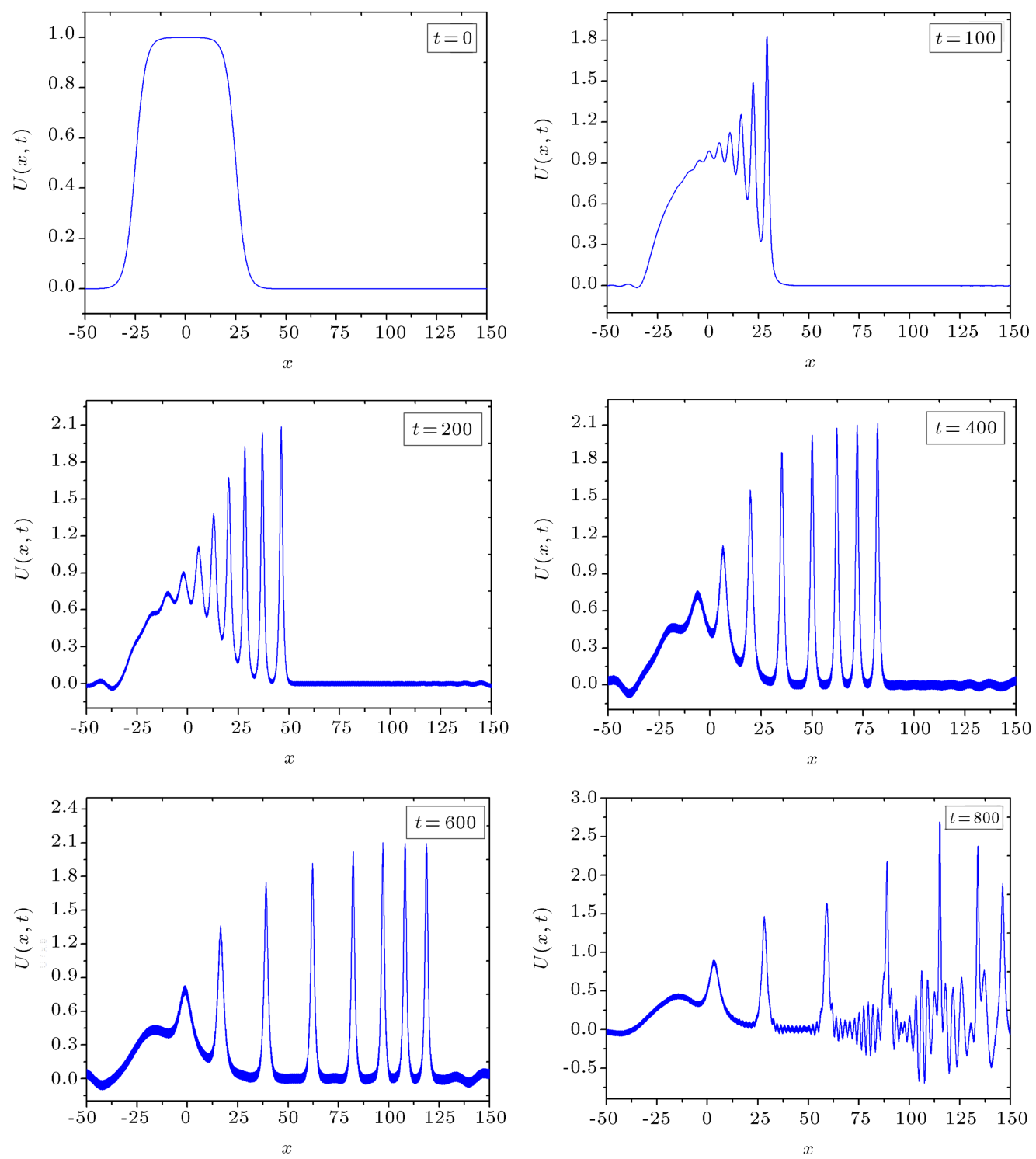

Figure 10. Developed train of solitons with $p=3, \varepsilon=0.2, \mu=0.1, c=0.3, h=0.1$, and $\Delta t=0.05$ at selected times.

visit in 2016. The fifth author (Seithuti Philemon Moshokoa) would like to thank the research support provided by the Department of Mathematics and Statistics at Tshwane University of Technology and the support from the South African National Foundation under Grant Number 92052 IRF1202210126.

\section{References}

1. Jawad, A.J.M., Petkovic, M.D., Laketa, P., and Biswas, A. "Dynamics of shallow water waves with Boussinesq equation", Scientia Iranica, Trans. B, 20(1), pp. 179-184 (2013).

2. Karakoc, S.B.G., Zeybek, H. and Ak, T. "Numerical solutions of the Kawahara equation by the septic Bspline collocation method", Statistics, Optimization and Information Computing, 2, pp. 211-221 (2014).
3. Girgis, L., Zerrad, E. and Biswas, A. "Solitary wave solutions of the Peregrine equation", International Journal of Oceans and Oceanography, 4(1), pp. 45-54 (2010).

4. Biswas, A. "1-Soliton solution of Benjamin-BonaMahony equation with dual-power law nonlinearity", Communications in Nonlinear Science and Numerical Simulation, 15(10), pp. 2744-2746 (2010).

5. Saka, B. "Quintic B-spline collocation method for numerical solution of the RLW equation", The ANZIAM Journal, 49(3), pp. 389-410 (2008).

6. Korkmaz, A. "Numerical algorithms for solutions of Korteweg-de Vries equation", Numerical Methods for Partial Differential Equations, 26(6), pp. 1504-1521 (2009).

7. Biswas, A., Krishnan, E.V., Suarez, P., Kara, A.H. and Kumar, S. "Solitary waves and conservation law 
of Bona-Chen equation", Indian Journal of Physics, 87(2), pp. 169-175 (2013).

8. Triki, H., Kara, A.H., Bhrawy, A., and Biswas, A. "Soliton solution and conservation law of GearGrimshaw model for shallow water waves", Acta Physica Polonica A, 125(5), pp. 1099-1106 (2014).

9. Osman, M.S. "Multi-soliton rational solutions for quantum Zakharov-Kuznetsov equation in quantum magnetoplasmas", Waves in Random and Complex Media, 26(4), pp. 434-443 (2016).

10. Osman, M.S. "Multiwave solutions of time-fractional $(2+1)$-dimensional Nizhnik Novikov Veselov equations", Pramana Journal of Physics, 88(4), 67 (2017).

11. Osman, M.S. "Nonlinear interaction of solitary waves described by multi-rational wave solutions of the $(2+$ 1)-dimensional Kadomtsev-Petviashvili equation with variable coefficients", Nonlinear Dynamics, 87(2), pp. 1209-1216 (2017).

12. Osman, M.S. "Analytical study of rational and doublesoliton rational solutions governed by the $\mathrm{KdV}$ Sawada-Kotera-Ramani equation with variable coefficients", Nonlinear Dynamics, 89(3), pp. 2283-2289 (2017).

13. Youssri, Y.H. "A new operational matrix of Caputo fractional derivatives of Fermat polynomials: an application for solving the Bagley-Torvik equation", Advances in Difference Equations, 73, pp. 1-17 (2017).

14. Sohail, A., Siddiqui, A.M. and Iftikhar, M. "Travelling wave solutions for fractional order KdV-like equations using $G^{\prime} / G$-expansion", Nonlinear Science Letters A, 8(2), pp. 228-235 (2017).

15. Sohail, A., Rees, J.M. and Zimmerman, W.B. "Analysis of capillary-gravity waves using the discrete periodic inverse scattering transform", Colloids and Surfaces A: Physicochemical and Engineering Aspects, 391(1), pp. 42-50 (2011).

16. Zeybek, H. and Karakoc, S.B.G. "A numerical investigation of the GRLW equation using lumped Galerkin approach with cubic B-spline", SpringerPlus, 5(199), pp. 1-17 (2016).

17. Ak, T., Karakoc, S.B.G. and Biswas, A. "A new approach for numerical solution of modified Kortewegde Vries equation", Iranian Journal of Science and Technology, Transactions A: Science (2017). doi.org/10.1007/s40995-017-0238-5

18. Ak, T., Karakoc, S.B.G., and Biswas, A. "Application of Petrov-Galerkin method to shallow water waves model: Modified Korteweg-de Vries equation", Scientia Iranica B, 24(3), pp. 1148-1159 (2017).

19. Karakoc, S.B.G. and Zeybek, H. "Solitary wave solutions of the GRLW equation using septic B-spline collocation method", Applied Mathematics and Computation, 289, pp. 159-171 (2016).

20. Triki, H., Ak, T., Moshokoa, S.P., and Biswas, A. "Soliton solutions to KdV equation with spatiotemporal dispersion", Ocean Engineering, 114, pp. 192-203 (2016).
21. Yagmurlu, N.M., Tasbozan, O., Ucar, Y., and Esen, A. "Numerical solutions of the combined KdV-mKdV equation by a quintic B-spline collocation method", Applied Mathematics \& Information Sciences Letters, 4(1), pp. 19-24 (2016).

22. Ak, T., Triki, H., and Biswas, A. "Numerical simulation for treatment of dispersive shallow water waves with Rosenau-KdV equation", The European Physical Journal Plus, 131(10), pp. 356-370 (2016).

23. Triki, H., Ak, T., and Biswas, A. "New types of solitonlike solutions for a second order wave equation of Korteweg-de Vries type", Applied and Computational Mathematics, 16(2), pp. 168-176 (2017).

24. Triki, H., Ak, T., Ekici, M., Sonmezoglu, A., Mirzazadeh, M., Kara, A.H., and Aydemir, T. "Some new exact wave solutions and conservation laws of potential Korteweg-de Vries equation", Nonlinear Dynamics, 89(1), pp. 501-508 (2017).

25. Abd-Elhameed, W.M. and Youssri, Y.H. "Spectral solutions for fractional differential equations via a novel Lucas operational matrix of fractional derivatives", Romanian Journal of Physics, 61(5-6), pp. 795-813 (2016).

26. Abd-Elhameed, W.M. and Youssri, Y.H. "Generalized Lucas polynomial sequence approach for fractional differential equations", Nonlinear Dynamics, 89(2), pp. 1341-1355 (2017).

27. Marchant, T.R. "Asymptotic solitons for a higherorder modified Korteweg-de Vries equation", Physical Review E, 66(046623), pp. 1-8 (2002).

28. Marchant, T.R. "Asymptotic solitons for a thirdorder Korteweg-de Vries equation", Chaos, Solitons \& Fractals, 22, pp. 261-270 (2004).

29. Jeffrey, A. and Kakutani, T. "Weak nonlinear dispersive waves: A discussion centered around the Korteweg-de Vries equation", SIAM Review, 14(4), pp. 582-643 (1972).

30. Canivar, A., Sari, M., and Dag, I. "A Taylor-Galerkin finite element method for the $\mathrm{KdV}$ equation using $\mathrm{cu}-$ bic B-splines", Physica B, 405, pp. 3376-3383 (2010).

31. Dag, I. and Dereli, Y. "Numerical solutions of KdV equation using radial basis functions", Applied Mathematical Modelling, 32(4), pp. 535-546 (2008).

32. Saka, B. "Cosine expansion-based differential quadrature method for numerical solution of the $\mathrm{KdV}$ equation", Chaos, Solitons \& Fractals, 40(5), pp. 2181-2190 (2009).

33. Ersoy, O. and Dag, I. "The exponential cubic B-spline algorithm for Korteweg-de Vries equation", Advances in Numerical Analysis, 2015, Article ID 367056, 8 pages (2015). 
34. Soliman, A.A. "Collocation solution of the Kortewegde Vries equation using septic splines", International Journal of Computer Mathematics, 81(3), pp. 325-331 (2004).

35. Zaki, S.I. "A quintic B-spline finite elements scheme for the KdVB equation", Computer Methods in Applied Mechanics and Engineering, 188, pp. 121-134 (2000).

36. Antonova, M. and Biswas, A. "Adiabatic parameter dynamics of perturbed solitary waves", Communications in Nonlinear Science and Numerical Simulation, 14(3), pp. 734-748 (2009).

37. Suli, A. and Mayers D.F., An Introduction to Numerical Analysis, Cambridge University Press, Cambridge, England (2003).

38. Prenter, P.M., Splines and Variational Methods, John Wiley, New York, USA (1975).

39. Bochev, P.B. and Gunzburger, M.D., Least-Squares Finite Element Methods, Springer, New York, USA (2009).

40. Thomee, V., Galerkin Finite Element Methods for Parabolic Problems, Springer, Berlin, Germany (2006).

41. Dhawan, S., Bhowmik, S.K., and Kumar, S. "Galerkinleast square B-spline approach toward advectiondiffusion equation", Applied Mathematics and Computation, 261, pp. 128-140 (2015).

42. Berezin, Y.A. and Karpman, V.I. "Nonlinear evolution of disturbances in plasma and other dispersive media", Soviet Physics JETP, 24, pp. 1049-1056 (1967).

\section{Biographies}

Turgut $\mathbf{A k}$ is a graduate student in the Department of Applied Mathematics at Nevsehir Haci Bektas Veli University, Turkey. He earned his BSc degree in Mathematics from Gazi University, Turkey. Subsequently, he obtained his MSc degree in Applied Mathematics from Erciyes University, Turkey. Then, he received PhD degree in Applied Mathematics from Nevsehir Hacı Bektaş Veli University, Turkey. Currently, he is working as a Lecturer of Mathematics in Armutlu Vocational School at Yalova University, Turkey. His current research interests are numerical analysis, computational methods, and wave models.

Houria Triki earned her PhD degree in Physics from Annaba University. Currently, she is a Faculty Member in Radiation Physics Laboratory at Badji Mokhtar University in Annaba, Algeria. She has published more than 90 papers in various peer-reviewed international journals of high repute and high impact factor. She has also participated in more than 60 scientific meetings.

Sharanjeet is an Assistant Professor in the Department of Mathematics at Central University of Haryana.
She received her $\mathrm{PhD}$ degree from NIT Jalandhar, Punjab, and worked as postdoc fellow with National Board of Higher Mathematics in the Department of Atomic Energy, Government of India. Her research interests are differential equations, numerical analysis, finite element methods, wavelets, etc.

Samir Kumar Bhowmik is working as an Associate Professor in the Department of Mathematics, Faculty of Science, University of Dhaka, Bangladesh. He earned his BSc and MSc degrees in Mathematics from Dhaka University. Then, he earned PhD degree in Mathematics from Heriot-Watt University, Edinburgh, UK, and post-doc from Korteweg-de Vries Institute for Mathematics, University of Amsterdam, Netherlands. He also worked as a visiting Faculty Member in the Department of Mathematics, IMSIU, Kingdom of Saudi Arabia, from August, 2013 to May, 2016. His research interest includes mathematical and numerical analysis, mathematical biology, computational finance, scientific computing, wave propagation, seismic imaging, and numerical linear algebra.

Seithuti Philemon Moshokoa earned his PhD degree in Mathematical Sciences from University of South Africa. He is working as a Professor in the Department of Mathematics and Statistics at Tshwane University of Technology in Pretoria, South Africa. His research interests are in extension and completion of mathematical structures, classical analysis, and topology.

Malik Zaka Ullah earned his PhD degree in Mathematics from University of Insubria, Italy. $\mathrm{He}$ is working as a lecturer in Department of Mathematics at King Khalid University in Abha, Saudi Arabia. His research interests are on computational mathematics, data communications, and network.

Anjan Biswas earned his BSc degree (with Honors) from Saint Xaviers College in Calcutta, India. Subsequently, he received his MSc and MPhil degrees in Applied Mathematics from University of Calcutta. Then, he earned MA and PhD degrees in Applied Mathematics from the University of New Mexico in Albuquerque, New Mexico, USA. Afterwards, he carried out his post-doctoral studies in Applied Mathematics at the University of Colorado, Boulder, USA. He was an Assistant Professor of Mathematics at Tennessee State University located in Nashville, TN, and Associate Professor of Mathematics at Delaware State University located in Dover, DE. Currently, he is working as a Professor of Mathematics at King Khalid University in Abha. His research interest is in theory of solitons. 